\title{
Applications of New Double Integral Transform (Laplace- Sumudu Transform) in Mathematical Physics
}

\author{
Shams A. Ahmed $\mathbb{D}^{1,2}$ \\ ${ }^{1}$ Department of Mathematic, Faculty of Sciences and Arts, Jouf University, Tubarjal, Saudi Arabia \\ ${ }^{2}$ Department of Mathematic, University of Gezira, Sudan \\ Correspondence should be addressed to Shams A. Ahmed; shamsalden20@hotmail.com
}

Received 18 October 2020; Revised 16 January 2021; Accepted 9 February 2021; Published 1 March 2021

Academic Editor: Alberto Fiorenza

Copyright (C) 2021 Shams A. Ahmed. This is an open access article distributed under the Creative Commons Attribution License, which permits unrestricted use, distribution, and reproduction in any medium, provided the original work is properly cited.

The primary purpose of this research is to demonstrate an efficient replacement of double transform called the double LaplaceSumudu transform (DLST) and prove some related theorems of the new double transform. Also, we will discuss the fundamental properties of the double Laplace-Sumudu transform of some basic functions. Then, by utilizing those outcomes, we will apply it to the partial differential equations to show its simplicity, efficiency, and high accuracy.

\section{Introduction}

Partial differential equations are used to describe many realworld problems arising in all the fields of applied science and issues associated with engineering. Due to the rapid advancement in science and engineering, various integral transforms have been used to solve the differential and integral equations, for instance, the Laplace transform [1], the Sumudu transform [2], the Elzaki transform [3], the natural transform [4], and many other double integral transforms $[5,6]$. However, most of the existing integral transforms have some limitations and cannot be used directly to solve nonlinear problems or many complex mathematical models. As a result, some researchers have combined these integral transforms with other methods such as the differential transform method, homotophy perturbation method, Adomian decomposition method and variational iteration method [7-13] for solving many nonlinear differential equations.

The usefulness of these equations has attracted the attention of many scholars throughout the history of applied sciences. One of the problems with these equations is that they are very difficult to solve equations with unknown functions of two variables sometimes. For this reason, this problem was solved by combining the Laplace transforms and Sumudu transform to give another double transform which is called the double Laplace-Sumudu transform.

In the present research, we consider linear, one-dimensional, time-dependent partial differential equation (PDE) of the form

$$
\sum_{n=0}^{N} c_{n} \frac{\partial^{n} \phi(x, t)}{\partial t^{n}}=\sum_{m=1}^{M} d_{m} \frac{\partial^{m} \phi(x, t)}{\partial x^{m}}+g(x, t),(\mathrm{x}, \mathrm{t}) \in \mathbb{R}_{+}^{2}
$$

with the initial conditions:

$$
\frac{\partial^{n} \phi(x, 0)}{\partial t^{n}}=f_{n}(x), n=0,1, \cdots, N-1, \mathrm{x} \in \mathbb{R}_{+}
$$

and boundary conditions:

$$
\frac{\partial^{m} \phi(0, t)}{\partial x^{m}}=h_{m}(t), m=0,1, \cdots, M-1, \mathrm{t} \in \mathbb{R}_{+},
$$

where $c_{n}, 0 \leq n \leq N ; d_{m}, 1 \leq m \leq M$ are given coefficients and $N, M$ are positive integers and $g(x, t)$ is the source term. 
The main objective of this paper is to develop new applications of the double Laplace-Sumudu transform for solving linear partial differential equations of the type (1) subject to the initial conditions (2) and boundary conditions (3). The proposed integral transform is successfully applied to a wide range of linear partial differential equations in mathematical physics.

\section{Preliminaries}

Definition 1. The double Laplace-Sumudu transform of the function $\phi(x, t)$ of two variables $x>0$ and $t>0$ is denoted by $L_{x} S_{t}[\phi(x, t)]=\bar{\phi}(\rho, \sigma)$ and defined as

$$
L_{x} S_{t}[\phi(x, t)]=\bar{\phi}(\rho, \sigma)=\frac{1}{\sigma} \int_{0}^{\infty} \int_{0}^{\infty} e^{-\rho x-(t / \sigma)} \phi(x, \mathrm{t}) d x d t
$$

whenever that integral exists. Here, $\rho$ and $\sigma$ are complex numbers.

Clearly, the double Laplace-Sumudu transform is a linear integral transformation as shown below:

$$
\begin{aligned}
& L_{x} S_{t}[\gamma \phi(x, t)+\eta \psi(x, t)]=\frac{1}{\sigma} \int_{0}^{\infty} \int_{0}^{\infty} e^{-\rho x-(t / \sigma)}[\gamma \phi(x, t)+\eta \psi(x, t)] d x d t \\
& \quad=\frac{1}{\sigma} \int_{0}^{\infty} \int_{0}^{\infty} e^{-\rho x-(t / \sigma)} \gamma \phi(x, t) d x d t+\frac{1}{\sigma} \int_{0}^{\infty} \int_{0}^{\infty} e^{-\rho x-(t / \sigma)} \eta \psi(x, t) d x d t \\
& \quad=\frac{\gamma}{\sigma} \int_{0}^{\infty} \int_{0}^{\infty} e^{-\rho x-(t / \sigma)} \phi(x, t) d x d t+\frac{\eta}{\sigma} \int_{0}^{\infty} \int_{0}^{\infty} e^{-\rho x-(t / \sigma)} \psi(x, t) d x d t \\
& =\gamma L_{x} S_{t}[\phi(x, t)]+\eta L_{x} S_{t}[\psi(x, t)],
\end{aligned}
$$

where $\gamma$ and $\eta$ are constants.

Definition 2. The inverse double Laplace-Sumudu transform $L_{x}^{-1} S_{t}^{-1}[\bar{\phi}(\rho, \sigma)]=\phi(x, t)$ is defined by the following form:

$L_{x}^{-1} S_{t}^{-1}[\bar{\phi}(\rho, \sigma)]=\phi(x, t)=\left(\frac{1}{2 \pi i}\right) \int_{\gamma-i \infty \infty}^{\gamma+i \infty} e^{\rho x} d \rho\left(\frac{1}{2 \pi i}\right) \int_{\eta-i \infty \infty}^{\eta+i \infty} \frac{1}{\sigma} e^{t / \sigma} \bar{\phi}(\rho, \sigma) d \sigma$.

Definition 3. The double Laplace-Sumudu transform formulas for the partial derivatives of an arbitrary integer order are given by (see the proof in Section 3.2)

$$
\begin{aligned}
& L_{x} S_{t}\left[\frac{\partial^{n} \phi(x, t)}{\partial t^{n}}\right]=\sigma^{-n} \bar{\phi}(\rho, \sigma)-\sum_{j=0}^{n-1} \sigma^{-n+j} L_{x}\left[\frac{\partial^{j} \phi(x, 0)}{\partial t^{j}}\right], \\
& L_{x} S_{t}\left[\frac{\partial^{m} \phi(x, t)}{\partial x^{m}}\right]=\rho^{m} \bar{\phi}(\rho, \sigma)-\sum_{k=0}^{m-1} \rho^{m-1-k} S_{t}\left[\frac{\partial^{k} \phi(0, t)}{\partial x^{k}}\right] .
\end{aligned}
$$

\section{Fundamental Properties of Double Laplace- Sumudu Transform}

3.1. Existence Condition for the Double Laplace-Sumudu Transform. If $\phi(x, t)$ is an exponential order $c$ and $d$ as $x \rightarrow$ $\infty, t \rightarrow \infty$., if there exists a positive constant $K$ such that $\forall$ $x>X, t>T$

$$
|\phi(x, t)|=K e^{c x+d t},
$$

and we write

$$
\phi(x, t)=O e^{c x+d t} \text { as } x \rightarrow \infty, t \rightarrow \infty .
$$

Or, equivalently,

$\lim _{x \rightarrow \infty, t \rightarrow \infty} e^{-\rho x-t / \sigma}|\phi(x, t)|=K \lim _{x \rightarrow \infty, t \rightarrow \infty} e^{-(\rho-c) x-(1 / \sigma-d) t}=0, \rho>c, \frac{1}{\sigma}>d$.

The function $\phi(x, t)$ is called an exponential order as $x$ $\rightarrow \infty, t \rightarrow \infty$, and clearly, it does not grow faster than $K$ $e^{c x+d t}$ as $x \rightarrow \infty, t \rightarrow \infty$.

Theorem 4. If a function $\phi(x, t)$ is a continuous function in every finite interval $(0, X)$ and $(0, T)$ of exponential order $e^{c x+d t}$, then the double Laplace-Sumudu transform of $\phi(x$ , $t$ ) exists for all $\rho$ and $1 / \sigma$ provided $\operatorname{Re}[\rho]>c$ and $\operatorname{Re}[1 /$ $\sigma]>d$.

Proof. From Definition 1, we have

$$
\begin{aligned}
|\bar{\phi}(\rho, \sigma)| & =\left|\frac{1}{\sigma} \int_{0}^{\infty} \int_{0}^{\infty} e^{-\rho x-(t / \sigma)} \phi(x, t) d x d t\right| \\
& \leq K \int_{0}^{\infty} e^{-(\rho-c) x} d x \int_{0}^{\infty} \frac{1}{\sigma} e^{-((t / \sigma)-d) t} d t \\
= & \frac{K}{(\rho-c)(1-d \sigma)}, \operatorname{Re}[\rho]>c, \operatorname{Re}\left[\frac{1}{\sigma}\right]>d .
\end{aligned}
$$

Then, from Equation (11), we have

$$
\lim _{x \rightarrow \infty, t \rightarrow \infty}|\bar{\phi}(\rho, \sigma)|=0 \text {, or } \lim _{x \rightarrow \infty, t \rightarrow \infty} \bar{\phi}(\rho, \sigma)=0 .
$$

3.2. Basic Derivative Properties of the Double LaplaceSumudu Transform. If $\bar{\phi}(\rho, \sigma)=L_{x} S_{t}[\phi(x, t)]$, then

$$
\begin{gathered}
(I) L_{x} S_{t}\left[\frac{\partial \phi(x, t)}{\partial x}\right]=\rho \bar{\phi}(\rho, \sigma)-S[\phi(0, t)], \\
(I I) L_{x} S_{t}\left[\frac{\partial \phi(x, t)}{\partial t}\right]=\frac{1}{\sigma} \bar{\phi}(\rho, \sigma)-\frac{1}{\sigma} L(\phi(x, 0)), \\
(I I I) L_{x} S_{t}\left[\frac{\partial^{2} \phi(x, t)}{\partial x^{2}}\right]=\rho^{2} \bar{\phi}(\rho, \sigma)-\rho S(\phi(0, t))-S\left(\phi_{x}(0, t)\right),
\end{gathered}
$$




$$
\begin{aligned}
& (I V) L_{x} S_{t}\left[\frac{\partial^{2} \phi(x, t)}{\partial t^{2}}\right]=\frac{1}{\sigma^{2}} \bar{\phi}(\rho, \sigma)-\frac{1}{\sigma^{2}} L(\phi(x, 0))-\frac{1}{\sigma} L\left(\phi_{t}(x, 0)\right), \\
& (V) L_{x} S_{t}\left[\frac{\partial^{2} \phi(x, t)}{\partial x \partial t}\right]=\frac{\rho}{\sigma} \bar{\phi}(\rho, \sigma)-\frac{\rho}{\sigma} L(\phi(x, 0))-S\left(\phi_{t}(0, t)\right) .
\end{aligned}
$$

Proof.

$$
\begin{aligned}
(I) L_{x} S_{t}\left[\frac{\partial \phi(x, t)}{\partial x}\right] & =\frac{1}{\sigma} \int_{0}^{\infty} \int_{0}^{\infty} e^{-\rho x-t / \sigma} \frac{\partial \phi(x, t)}{\partial x} d x d t \\
& =\frac{1}{\sigma} \int_{0}^{\infty} e^{-t / \sigma} d t\left\{\int_{0}^{\infty} e^{-\rho x} \frac{\partial \phi(x, t)}{\partial x} d x\right\}
\end{aligned}
$$

let $u=e^{-\rho x}, d v=(\partial \phi(x, t) / \partial x) d x$, thus

$$
\begin{aligned}
L_{x} S_{t}\left[\frac{\partial \phi(x, t)}{\partial x}\right] & =\frac{1}{\sigma} \int_{0}^{\infty} e^{-t / \sigma} d t\left\{-\phi(0, t)+\rho \int_{0}^{\infty} e^{-\rho x} \phi(x, t) d x\right\} \\
& =\rho \bar{\phi}(\rho, \sigma)-S(\phi(0, t)) . \\
(I I) L_{x} S_{t}\left[\frac{\partial \phi(x, t)}{\partial t}\right] & =\frac{1}{\sigma} \int_{0}^{\infty} \int_{0}^{\infty} e^{-\rho x-t / \sigma} \frac{\partial \phi(x, t)}{\partial t} d x d t \\
& =\frac{1}{\sigma} \int_{0}^{\infty} e^{-\rho x} d x\left\{\int_{0}^{\infty} e^{-t / \sigma} \frac{\partial \phi(x, t)}{\partial t} d t\right\}
\end{aligned}
$$

let $u=e^{-t / \sigma}, d v=\partial \phi(x, t) / \partial t d t$, then

$$
\begin{aligned}
L_{x} S_{t}\left[\frac{\partial \phi(x, t)}{\partial t}\right] & =\frac{1}{\sigma} \int_{0}^{\infty} e^{-\rho x} d x\left\{-\phi(x, 0)+\frac{1}{\sigma} \int_{0}^{\infty} e^{-t / \sigma} \phi(x, t) d t\right\} \\
& =\frac{1}{\sigma} \bar{\phi}(\rho, \sigma)-\frac{1}{\sigma} L(\phi(x, 0)) .
\end{aligned}
$$

Similarly, we can prove that (III), (IV), and (V).

\section{Double Laplace-Sumudu Transform (DLST)}

Applying the double Laplace-Sumudu transform on both sides of (1), we get

$$
\begin{aligned}
\sum_{n=0}^{N} c_{n} & {\left[\sigma^{-n} \bar{\phi}(\rho, \sigma)-\sum_{j=0}^{n-1} \sigma^{-n+j} L_{x}\left[\frac{\partial^{j} \phi(x, 0)}{\partial t^{j}}\right]\right] } \\
= & \sum_{m=1}^{M} d_{m}\left[\rho^{m} \bar{\phi}(\rho, \sigma)-\sum_{k=0}^{m-1} \rho^{m-1-k} S_{t}\left[\frac{\partial^{k} \phi(0, t)}{\partial x^{k}}\right]\right] \\
+ & \bar{g}(\rho, \sigma)
\end{aligned}
$$

Further, applying single Laplace transform (LT) to initial (2) and single Sumudu transform (ST) to boundary (3), we get

$$
\begin{aligned}
L_{x}\left[\frac{\partial^{n} \phi(x, 0)}{\partial t^{n}}\right] & =\overline{f_{n}}(\rho), n=0,1, \cdots, N-1, S_{t}\left[\frac{\partial^{m} \phi(0, t)}{\partial x^{m}}\right] \\
& =\overline{h_{m}}(\sigma), m=0,1, \cdots, M-1,
\end{aligned}
$$

substituting (18) in (17) and simplifying, we obtain

$$
\begin{aligned}
& \sum_{n=0}^{N} c_{n}\left[\sigma^{-n} \bar{\phi}(\rho, \sigma)-\sum_{j=0}^{n-1} \sigma^{-n+j} \overline{f_{n}}(\rho)\right] \\
& \quad=\sum_{m=1}^{M} d_{m}\left[\rho^{m} \bar{\phi}(\rho, \sigma)-\sum_{k=0}^{m-1} \rho^{m-1-k} \overline{h_{m}}(\sigma)\right]+\bar{g}(\rho, \sigma) .
\end{aligned}
$$

Equation (19) is an algebraic equation in $\bar{\phi}(\rho, \sigma)$. Solving this algebraic equation and taking $L_{x}^{-1} S_{t}^{-1}[\bar{\phi}(\rho, \sigma)]$, we obtain an exact solution $\phi(x, t)$ of Equation (1).

\section{Elucidative Examples}

In this section, the applications of the proposed transform are presented. The simplicity, efficiency, and high accuracy of the double Laplace-Sumudu transform are clearly illustrated.

5.1. The Advection-Diffusion Equation. By substituting $N=$ $1, M=2, c_{0}=g=0, c_{1}=1$ in (1), we have got the advectiondiffusion equation:

$$
\frac{\partial \phi(x, t)}{\partial t}=d_{2} \frac{\partial^{2} \phi(x, t)}{\partial x^{2}}+d_{1} \frac{\partial \phi(x, t)}{\partial x}
$$

with ICs and BCs

$$
\begin{gathered}
\phi(x, 0)=f_{0}(x), \\
\phi(0, t)=h_{0}(t), \\
\phi_{x}(0, t)=h_{1}(t),
\end{gathered}
$$

then (19) gives the solution of (20) as

$\phi(x, t)=L_{x}^{-1} S_{t}^{-1}\left[\frac{1}{\left(1-d_{2} \sigma \rho^{2}-d_{1} \sigma \rho\right)}\left[\overline{f_{0}}(\rho)-d_{2} \sigma \rho \overline{h_{0}}(\sigma)-d_{2} \sigma \overline{h_{1}}(\sigma)-d_{1} \sigma \overline{h_{0}}(\sigma)\right]\right]$.

Example 5. Putting $d_{1}=-1, d_{2}=1$ in (20), we got

$$
\frac{\partial \phi(x, t)}{\partial t}=\frac{\partial^{2} \phi(x, t)}{\partial x^{2}}-\frac{\partial \phi(x, t)}{\partial x}
$$

with the conditions,

$$
\begin{gathered}
\phi(x, 0)=e^{x}-x=f_{0}(x), \\
\phi(0, t)=1+t=h_{0}(t), \\
\phi_{x}(0, t)=0=h_{1}(t) .
\end{gathered}
$$


TABLE 1: The double Laplace-Sumudu transform of some basic functions.

\begin{tabular}{lcr}
\hline Sr. no. & $\phi(x, t)$ & $L_{x} S_{t}[\phi(x, t)]=\bar{\phi}(\rho, \sigma)$ \\
\hline 1 & 1 & $1 / \rho$ \\
2 & $x^{c} t^{d}$, if $c$ and $d$ are positive integral & $\left(c ! d ! / \rho^{c+1}\right) \sigma^{d}$ \\
3 & $e^{c x+d t}$ & $1 /(\rho-c)(1-d \sigma)$ \\
4 & $\sin (c x+d t)$ & $c+d \sigma \rho /\left(\rho^{2}+c^{2}\right)\left(1+d^{2} \sigma^{2}\right)$ \\
5 & $\cos (c x+d t)$ & $\rho-c d \sigma /\left(\rho^{2}+c^{2}\right)\left(1+d^{2} \sigma^{2}\right)$ \\
6 & $\sinh (c x+d t)$ & $c+d \sigma \rho /\left(\rho^{2}-c^{2}\right)\left(1-d^{2} \sigma^{2}\right)$ \\
7 & $\cosh (c x+d t)$ & $\rho+c d \sigma /\left(\rho^{2}-c^{2}\right)\left(1-d^{2} \sigma^{2}\right)$ \\
8 & $J_{0}(c \sqrt{x t})$, where $J_{0}(x)$ is the modified Bessel function of order zero & $4 / 4 \rho+\sigma c^{2}$ \\
9 & $\phi(x-\delta, t-\varepsilon) H(x-\delta, t-\varepsilon)$, where $H(x, t)$ is the Heaviside unit step function & $e^{-\rho \delta-(\varepsilon / \sigma)} \bar{\phi}(\rho, \sigma)$ \\
10 & $(\phi * * \psi)(x, t)($ convolution theorem $)$ & $\sigma \bar{\phi}(\rho, \sigma) \bar{\psi}(\rho, \sigma)$ \\
11 & $f(x) g(t)$ & $L_{x}[f(x)] S_{t}[g(t)]$ \\
\hline
\end{tabular}

Substituting

$$
\begin{gathered}
\overline{f_{0}}(\rho)=\frac{1}{\rho-1}-\frac{1}{\rho^{2}}, \\
\overline{h_{0}}(\sigma)=1+\sigma, \\
\overline{h_{1}}(\sigma)=0,
\end{gathered}
$$

in (22) and simplifying, we get a solution of (23)

$$
\phi(x, t)=L_{x}^{-1} S_{t}^{-1}\left[\frac{1}{(\rho-1)}-\frac{1}{\rho^{2}}+\frac{\sigma}{\rho}\right]=e^{x}-x+t .
$$

5.2. The Reaction-Diffusion Equation. By substituting $N=1$, $M=2, g=0, c_{1}=1, d_{1}=0$, and $d_{2}>0$ in (1), we have the reaction-diffusion equation:

$$
c_{0} \phi(x, t)+\frac{\partial \phi(x, t)}{\partial t}=d_{2} \frac{\partial^{2} \phi(x, t)}{\partial x^{2}}
$$

with ICs and BCs:

$$
\begin{gathered}
\phi(x, 0)=f_{0}(x), \\
\phi(0, t)=h_{0}(t), \\
\phi_{x}(0, t)=h_{1}(t),
\end{gathered}
$$

then (19) gives the solution of (27) as

$$
\phi(x, t)=L_{x}^{-1} S_{t}^{-1}\left[\frac{1}{\left(1+c_{0} \sigma-d_{2} \sigma \rho^{2}\right)}\left[\overline{f_{0}}(\rho)-d_{2} \sigma \rho \overline{h_{0}}(\sigma)-d_{2} \sigma \overline{h_{1}}(\sigma)\right]\right] .
$$

5.2.1. The Heat (Diffusion) Equation. Taking $c_{0}=0$ in (27), we have the linear heat equation:

$$
\frac{\partial \phi(x, t)}{\partial t}=d_{2} \frac{\partial^{2} \phi(x, t)}{\partial x^{2}}
$$

with ICs and BCs:

$$
\begin{gathered}
\phi(x, 0)=f_{0}(x), \\
\phi(0, t)=h_{0}(t), \\
\phi_{x}(0, t)=h_{1}(t),
\end{gathered}
$$

then (29) gives the solution of (30) as

$$
\phi(x, t)=L_{x}^{-1} S_{t}^{-1}\left[\frac{1}{\left(1-d_{2} \sigma \rho^{2}\right)}\left[\overline{f_{0}}(\rho)-d_{2} \sigma \rho \overline{h_{0}}(\sigma)-d_{2} \sigma \overline{h_{1}}(\sigma)\right]\right] .
$$

Example 6. Putting $d_{2}=1$ in (30) to yield

$$
\frac{\partial \phi(x, t)}{\partial t}=\frac{\partial^{2} \phi(x, t)}{\partial x^{2}}
$$

with the conditions,

$$
\begin{gathered}
\phi(x, 0)=\sin x=f_{0}(x), \\
\phi(0, t)=0=h_{0}(t), \\
\phi_{x}(0, t)=e^{-t}=h_{1}(t) .
\end{gathered}
$$

Substituting

$$
\overline{f_{0}}(\rho)=\frac{1}{\rho^{2}+1}, \overline{h_{0}}(\sigma)=0, \overline{h_{1}}(\sigma)=\frac{1}{1+\sigma}
$$

in (32) and simplifying, we get a solution of (33)

$$
\phi(x, t)=L_{x}^{-1} S_{t}^{-1}\left[\frac{1}{\left(\rho^{2}+1\right)(1+\sigma)}\right]=e^{-t} \sin x .
$$

5.3. The Telegraph Equation. By substituting $N=M=2, d_{1}$ 
$=g=0$ in (1), we have got the linear telegraph equation

$$
c_{0} \phi(x, t)+c_{1} \frac{\partial \phi(x, t)}{\partial t}+c_{2} \frac{\partial^{2} \phi(x, t)}{\partial t^{2}}=d_{2} \frac{\partial^{2} \phi(x, t)}{\partial x^{2}},
$$

with ICs and BCs:

$$
\begin{gathered}
\phi(x, 0)=f_{0}(x), \\
\phi_{t}(x, 0)=f_{1}(x), \\
\phi(0, t)=h_{0}(t), \\
\phi_{x}(0, t)=h_{1}(t),
\end{gathered}
$$

then (19) gives the solution of (37) as

$$
\phi(x, t)=L_{x}^{-1} S_{t}^{-1}\left[\frac{1}{\left(c_{0} \sigma^{2}+c_{1} \sigma+c_{2}-d_{2} \sigma^{2} \rho^{2}\right)}\left[\begin{array}{c}
c_{1} \overline{f_{0}}(\rho)+c_{2} \overline{f_{0}}(\rho)+c_{2} \sigma \overline{f_{1}}(\rho) \\
-d_{2} \sigma^{2} \rho \overline{h_{0}}(\sigma)-d_{2} \sigma^{2} \overline{h_{1}}(\sigma)
\end{array}\right] .\right.
$$

5.3.1. The Wave Equation. Taking $c_{0}=c_{1}=0, c_{2}=1$ in (37), we obtain the linear wave equation:

$$
\frac{\partial^{2} \phi(x, t)}{\partial t^{2}}=d_{2} \frac{\partial^{2} \phi(x, t)}{\partial x^{2}}
$$

with ICs and BCs

$$
\begin{gathered}
\phi(x, 0)=f_{0}(x), \\
\phi_{t}(x, 0)=f_{1}(x), \\
\phi(0, t)=h_{0}(t), \\
\phi_{x}(0, t)=h_{1}(t),
\end{gathered}
$$

then (39) gives the solution of (40) as

$$
\phi(x, t)=L_{x}^{-1} S_{t}^{-1}\left[\frac{1}{\left(1-d_{2} \sigma^{2} \rho^{2}\right)}\left[\overline{f_{0}}(\rho)+\sigma \overline{f_{1}}(\rho)-d_{2} \sigma^{2} \rho \overline{h_{0}}(\sigma)-d_{2} \sigma^{2} \overline{h_{1}}(\sigma)\right]\right] .
$$

5.4. The Klein-Gordon Equation. By substituting $N=M=2$ , $c_{1}=d_{1}=0, c_{2}=1$ in (1), we have the Klein-Gordon equation:

$$
c_{0} \phi(x, t)+\frac{\partial^{2} \phi(x, t)}{\partial t^{2}}=d_{2} \frac{\partial^{2} \phi(x, t)}{\partial x^{2}}+g(x, t)
$$

with ICs and BCs

$$
\begin{gathered}
\phi(x, 0)=f_{0}(x), \\
\phi_{t}(x, 0)=f_{1}(x), \\
\phi(0, t)=h_{0}(t), \\
\phi_{x}(0, t)=h_{1}(t),
\end{gathered}
$$

then (19) gives the solution of (43) as

$$
\phi(x, t)=L_{x}^{-1} S_{t}^{-1}\left[\frac{1}{\left(1+c_{0} \sigma^{2}-d_{2} \sigma^{2} \rho^{2}\right)}\left[\begin{array}{c}
\overline{f_{0}}(\rho)+\sigma \overline{f_{1}}(\rho)-d_{2} \sigma^{2} \rho \overline{h_{0}}(\sigma) \\
-d_{2} \sigma^{2} \overline{h_{1}}(\sigma)+\sigma^{2} \bar{g}(\rho, \sigma)
\end{array}\right]\right]
$$

Example 7. Putting $c_{0}=-2, d_{2}=1$, and $g(x, t)=-2 \sin x$ $\sin t$ in (43) to yield

$$
\frac{\partial^{2} \phi(x, t)}{\partial t^{2}}-2 \phi(x, t)=\frac{\partial^{2} \phi(x, t)}{\partial x^{2}}-2 \sin x \sin t
$$

with the conditions,

$$
\begin{gathered}
\phi(x, 0)=0=f_{0}(x), \\
\phi_{t}(x, 0)=\sin x=f_{1}(x), \\
\phi(0, t)=0=h_{0}(t), \\
\phi_{x}(0, t)=\sin t=h_{1}(t) .
\end{gathered}
$$

Substituting

$$
\begin{gathered}
\overline{f_{0}}(\rho)=0, \\
\overline{f_{1}}(\rho)=\frac{1}{\rho^{2}+1}, \\
\overline{h_{0}}(\sigma)=0, \\
\overline{h_{1}}(\sigma)=\frac{\sigma}{1+\sigma^{2}}, \\
\bar{g}(\rho, \sigma)=\frac{-2 \sigma}{\left(\rho^{2}+1\right)\left(1+\sigma^{2}\right)},
\end{gathered}
$$

in (45) and simplifying, we get a solution of (46)

$$
\phi(x, t)=L_{x}^{-1} S_{t}^{-1}\left[\frac{\sigma}{\left(\rho^{2}+1\right)\left(1+\sigma^{2}\right)}\right]=\sin x \sin t
$$

5.5. The Dissipative Wave Equation. By substituting $N=M$ $=2, c_{0}=0, c_{2}=1$ in (1), we have the linear dissipative wave equation:

$$
c_{1} \frac{\partial \phi(x, t)}{\partial t}+\frac{\partial^{2} \phi(x, t)}{\partial t^{2}}=d_{2} \frac{\partial^{2} \phi(x, t)}{\partial x^{2}}+d_{1} \frac{\partial \phi(x, t)}{\partial x}+g(x, t),
$$

with ICs and BCs:

$$
\begin{gathered}
\phi(x, 0)=f_{0}(x), \\
\phi_{t}(x, 0)=f_{1}(x), \\
\phi(0, t)=h_{0}(t), \\
\phi_{x}(0, t)=h_{1}(t),
\end{gathered}
$$


then (19) gives the solution of (50) as

$$
\begin{aligned}
\phi(x, t)= & L_{x}^{-1} S_{t}^{-1}\left[\frac{1}{\left(1+c_{1} \sigma-d_{2} \sigma^{2} \rho^{2}-d_{1} \sigma^{2} \rho\right)}\right. \\
& \left.\cdot\left[\begin{array}{c}
c_{1} \sigma \overline{f_{0}}(\rho)+\overline{f_{0}}(\rho)+\sigma \overline{f_{1}}(\rho)-d_{2} \sigma^{2} \rho \overline{h_{0}}(\sigma) \\
-d_{2} \sigma^{2} \overline{h_{1}}(\sigma)-d_{1} \sigma^{2} \overline{h_{0}}(\sigma)+\sigma^{2} \bar{g}(\rho, \sigma)
\end{array}\right]\right] .
\end{aligned}
$$

Example 8. Putting $c_{1}=d_{1}=d_{2}=1$ and $g(x, t)=2(t-x)$ in (50) to yield

$$
\frac{\partial \phi(x, t)}{\partial t}+\frac{\partial^{2} \phi(x, t)}{\partial t^{2}}=\frac{\partial^{2} \phi(x, t)}{\partial x^{2}}+\frac{\partial \phi(x, t)}{\partial x}+2(t-x),
$$

with the conditions,

$$
\begin{aligned}
& \phi(x, 0)=x^{2}=f_{0}(x), \\
& \phi_{t}(x, 0)=0=f_{1}(x), \\
& \phi(0, t)=t^{2}=h_{0}(t), \\
& \phi_{x}(0, t)=0=h_{1}(t) .
\end{aligned}
$$

Substituting

$$
\begin{gathered}
\overline{f_{0}}(\rho)=\frac{2}{\rho^{3}}, \\
\overline{f_{1}}(\rho)=0, \\
\overline{h_{0}}(\sigma)=2 \sigma^{2}, \\
\overline{h_{1}}(\sigma)=0 \\
\bar{g}(\rho, \sigma)=2\left(\frac{\sigma}{\rho}-\frac{1}{\rho^{2}}\right),
\end{gathered}
$$

in (52) and simplifying, we get a solution of (53)

$$
\phi(x, t)=L_{x}^{-1} S_{t}^{-1}\left[\frac{2}{\rho^{3}}+\frac{2 \sigma^{2}}{\rho}\right]=x^{2}+t^{2} .
$$

5.6. The Korteweg-de Vries (KdV) Equation. By substituting $N=1, M=3, c_{0}=d_{2}=0, c_{1}=1$ in (1), we have the linear Korteweg-de Vries (KdV) equation:

$$
\frac{\partial \phi(x, t)}{\partial t}=d_{3} \frac{\partial^{3} \phi(x, t)}{\partial x^{3}}+d_{1} \frac{\partial \phi(x, t)}{\partial x}+g(x, t),
$$

with ICs and BCs

$$
\begin{gathered}
\phi(x, 0)=f_{0}(x), \\
\phi(0, t)=h_{0}(t), \\
\phi_{x}(0, t)=h_{1}(t), \\
\phi_{x x}(0, t)=h_{2}(t),
\end{gathered}
$$

then (19) gives the solution of (57) as

$$
\begin{aligned}
\phi(x, t)= & L_{x}^{-1} S_{t}^{-1}\left[\frac{1}{\left(1-d_{3} \sigma \rho^{3}-d_{1} \sigma \rho\right)}\right. \\
& \left.\cdot\left[\begin{array}{c}
\overline{f_{0}}(\rho)-d_{3} \sigma \rho^{2} \overline{h_{0}}(\sigma)-d_{3} \sigma \rho \overline{h_{1}}(\sigma) \\
-d_{3} \sigma \overline{h_{2}}(\sigma)-d_{1} \sigma \overline{h_{0}}(\sigma)+\sigma \bar{g}(\rho, \sigma)
\end{array}\right]\right] .
\end{aligned}
$$

Example 9. Putting $d_{3}=d_{1}=-1$ and $g(x, t)=0$ in (57) to yield

$$
\frac{\partial \phi(x, t)}{\partial t}=-\frac{\partial^{3} \phi(x, t)}{\partial x^{3}}-\frac{\partial \phi(x, t)}{\partial x}
$$

with the conditions,

$$
\begin{gathered}
\phi(x, 0)=e^{-x}=f_{0}(x), \\
\phi(0, t)=e^{2 t}=h_{0}(t), \\
\phi_{x}(0, t)=-e^{2 t}=h_{1}(t), \\
\phi_{x x}(0, t)=e^{2 t}=h_{2}(t) .
\end{gathered}
$$

Substituting

$$
\begin{gathered}
\overline{f_{0}}(\rho)=\frac{1}{\rho+1}, \\
\overline{h_{0}}(\sigma)=\overline{h_{2}}(\sigma)=\frac{1}{1-2 \sigma}, \\
\overline{h_{1}}(\sigma)=\frac{-1}{1-2 \sigma},
\end{gathered}
$$

in (59) and simplifying, we get a solution of (60)

$$
\phi(x, t)=L_{x}^{-1} S_{t}^{-1}\left[\frac{1}{(\rho+1)(1-2 \sigma)}\right]=e^{2 t-x} .
$$

Example 10. Putting $d_{3}=1, d_{1}=0$, and $g(x, t)=2 e^{t-x}$ in (57) to obtain the linear third-order dispersive, inhomogeneous equation:

$$
\frac{\partial \phi(x, t)}{\partial t}=\frac{\partial^{3} \phi(x, t)}{\partial x^{3}}+2 e^{t-x}
$$

with the conditions,

$$
\begin{gathered}
\phi(x, 0)=1+e^{-x}=f_{0}(x), \\
\phi(0, t)=1+e^{t}=h_{0}(t), \\
\phi_{x}(0, t)=-e^{t}=h_{1}(t), \\
\phi_{x x}(0, t)=e^{t}=h_{2}(t) .
\end{gathered}
$$


Substituting

$$
\begin{gathered}
\overline{f_{0}}(\rho)=\frac{1}{\rho}+\frac{1}{\rho+1}, \\
\overline{h_{0}}(\sigma)=1+\frac{1}{1-\sigma}, \\
\overline{h_{1}}(\sigma)=\frac{-1}{1-\sigma}, \\
\overline{h_{2}}(\sigma)=\frac{1}{1-\sigma}, \\
\bar{g}(\rho, \sigma)=\frac{2}{(\rho+1)(1-\sigma)},
\end{gathered}
$$

in (59) and simplifying, we get a solution of (64)

$$
\phi(x, t)=L_{x}^{-1} S_{t}^{-1}\left[\frac{1}{\rho}+\frac{1}{(\rho+1)(1-\sigma)}\right]=1+e^{t-x} .
$$

5.7. The Euler-Bernoulli Equation. By substituting $N=2, M$ $=4, c_{0}=c_{1}=d_{1}=d_{2}=d_{3}=0, c_{2}=-1$ in (1), we have the Euler-Bernoulli equation:

$$
-\frac{\partial^{2} \phi(x, t)}{\partial t^{2}}=d_{4} \frac{\partial^{4} \phi(x, t)}{\partial x^{4}}+g(x, t)
$$

with ICs and BCs:

$$
\begin{gathered}
\phi(x, 0)=f_{0}(x), \\
\phi_{t}(x, 0)=f_{1}(x), \\
\phi(0, t)=h_{0}(t), \\
\phi_{x}(0, t)=h_{1}(t), \\
\phi_{x x}(0, t)=h_{2}(t), \\
\phi_{x x x}(0, t)=h_{3}(t),
\end{gathered}
$$

then (19) gives the solution of (68) as

$$
\begin{aligned}
\phi(x, t)= & L_{x}^{-1} S_{t}^{-1}\left[\frac{1}{\left(1+d_{4} \sigma^{2} \rho^{4}\right)}\right. \\
& \left.\cdot\left[\begin{array}{c}
\overline{f_{0}}(\rho)+\sigma \overline{f_{1}}(\rho)+d_{4} \sigma^{2} \rho^{3} \overline{h_{0}}(\sigma)+d_{4} \sigma^{2} \rho^{2} \overline{h_{1}}(\sigma) \\
+d_{4} \sigma^{2} \rho \overline{h_{2}}(\sigma)+d_{4} \sigma^{2} \overline{h_{3}}(\sigma)-\sigma^{2} \bar{g}(\rho, \sigma)
\end{array}\right]\right] .
\end{aligned}
$$

Example 11. Putting $d_{4}=1$ and $g(x, t)=-x t-t^{2}$ in (68) to yield

$$
-\frac{\partial^{2} \phi(x, t)}{\partial t^{2}}=\frac{\partial^{4} \phi(x, t)}{\partial x^{4}}-x t-t^{2}
$$

with the conditions,

$$
\begin{gathered}
\phi(x, 0)=0=f_{0}(x), \\
\phi_{t}(x, 0)=\frac{x^{5}}{5 !}=f_{1}(x), \\
\phi(0, t)=\frac{t^{4}}{12}=h_{0}(t), \\
\phi_{x}(0, t)=0=h_{1}(t), \\
\phi_{x x}(0, t)=0=h_{2}(t), \\
\phi_{x x x}(0, t)=0=h_{3}(t),
\end{gathered}
$$

Substituting

$$
\begin{gathered}
\overline{f_{0}}(\rho)=0, \\
\overline{f_{1}}(\rho)=\frac{1}{\rho^{6}}, \\
\overline{h_{0}}(\sigma)=2 \sigma^{4}, \\
\overline{h_{1}}(\sigma)=\overline{h_{2}}(\sigma)=\overline{h_{3}}(\sigma)=0, \\
\bar{g}(\rho, \sigma)=\frac{-\sigma}{\rho^{2}}-\frac{2 \sigma^{2}}{\rho},
\end{gathered}
$$

in (70) and simplifying, we get a solution of (71)

$$
\phi(x, t)=L_{x}^{-1} S_{t}^{-1}\left[\frac{\sigma}{\rho^{6}}+\frac{2 \sigma^{4}}{\rho}\right]=\frac{x^{5} t}{5 !}+\frac{t^{4}}{12} .
$$

\section{Conclusion}

We introduced an efficient integral transform called the double Laplace-Sumudu transform to obtain exact solutions of linear partial differential equations. We presented its existence and inverse transform. Also, we presented some useful properties and theorems to seek out the solution of a wide range of linear partial differential equations. Finally, based on the mathematical formulations, simplicity, and the findings of the proposed integral transform, we conclude that it is highly efficient because of the following advantages:

(i) The single Laplace and Sumudu transform to solve equations with unknown functions of two variables were hard and useless sometimes. For this reason, this problem was solved by combining the Laplace transforms and Sumudu transform to give another double transform which is called the double Laplace-Sumudu transform

(ii) It can easily be applied directly to solve a wide range of linear partial differential equations in mathematical physics by turning these equations into algebraic ones

(iii) For advanced research in physical science and engineering, the proposed integral transform can be considered a stepping stone to solve a wide range of linear partial differential equations. 


\section{Data Availability}

No data were used to support this study.

\section{Conflicts of Interest}

The author declares that he has no conflict of interest.

\section{References}

[1] R. M. Spiegel, Theory and problems of Laplace transform, Schaum's Outline Series, McGraw - Hill, New York, USA, 1965.

[2] G. K. Weatugala, "Sumudu transforms - a new integral transform to solve differential equations and control engineering problems," Mathematic Engineering, vol. 61, pp. 319-329, 1993.

[3] T. M. Elzaki, "The new integral transform "Elzaki transform"," Global Journal of Pure and Applied Mathematics, vol. 7, pp. 57-64, 2011.

[4] Z. H. Khan and W. A. Khan, "N-transform-properties and applications," NUST Journal of Engineering Sciences, vol. 1, pp. 127-133, 2008.

[5] L. Debnath, "The double Laplace transforms and their properties with applications to functional, integral and partial differential equations," International Journal of Applied and Computational Mathematics, vol. 2, no. 2, pp. 223-241, 2016.

[6] S. Ahmed, T. Elzaki, and A. A. Hassan, "Solution of integral differential equations by new double integral transform (Laplace-Sumudu transform)," Journal Abstract and Applied Analysis, vol. 2020, pp. 1-7, 2020.

[7] S. Ahmed and T. Elzaki, "On the comparative study integro Differential equations using difference numerical methods," Journal of King Saud University - Science, vol. 32, no. 1, pp. 84-89, 2020.

[8] S. Ahmed, M. Elbadri, and M. Z. Mohamed, "A New Efficient Method for Solving Two-Dimensional Nonlinear System of Burger's Differential Equations," Journal Abstract and Applied Analysis, vol. 2020, article 7413859, pp. 1-7, 2020.

[9] H. Kumar Mishra and A. K. Nagar, "He-Laplace method for linear and nonlinear partial differential equations," Journal of Applied Mathematics, vol. 2012, Article ID 180315, 16 pages, 2012.

[10] A. E. Hamza and T. M. Elzaki, "Application of homotopy perturbation and Sumudu Transform Method for Solving Burgers Equations," American Journal of Theoretical and Applied Statistics, vol. 4, no. 6, pp. 480-483, 2015.

[11] E. Hilal and T. M. Elzaki, "Solution of nonlinear partial differential equations by new Laplace variational iteration method," Journal of Function Spaces, vol. 2014, Article ID 790714, 5 pages, 2014.

[12] M. Elbadri, S. A. Ahmed, Y. T. Abdalla, and W. Hdidi, "A new solution of time-fractional coupled $\mathrm{KdV}$ equation by using natural decomposition method," Journal Abstract and Applied Analysis, vol. 2020, article 3950816, 9 pages, 2020.

[13] S. Ahmed and M. Elbadri, "Solution of Newell -Whitehead -Segal equation of fractional order by using Sumudu decomposition method," Mathematics and Statistics, vol. 8, no. 6, pp. 631-636, 2020. 